International Journal of Current Advanced Research

ISSN: O: 2319-6475, ISSN: P: 2319 - 6505, Impact Factor: SJIF: 5.995

Available Online at www.journalijcar.org

Volume 6; Issue 5; May 2017; Page No. 3960-3963

DOI: http://dx.doi.org/10.24327/ijcar.2017.3963.0406

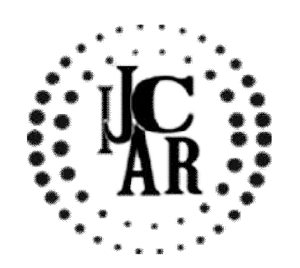

Research Article

\title{
A CROSS SECTIONAL STUDY OF FETAL OUTCOME IN CASES OF POLYHYDRAMNIOS AT TERTIARY CARE CENTER
}

\section{Neethika Raghuwanshi ${ }^{1}$ and Sumit Suresh Aggarwal ${ }^{2}$}

${ }^{1}$ Department of Obstetrics \& Gynecology, Govt Medical College, Akola

${ }^{2}$ Department of Community Medicine, Govt Medical College, Akola

\section{A R T I C L E I N F O}

\section{Article History:}

Received $22^{\text {nd }}$ February, 2017

Received in revised form $17^{\text {th }}$ March, 2017

Accepted $8^{\text {th }}$ April, 2017

Published online $28^{\text {th }}$ May, 2017

\section{Key words:}

Polyhydramnios, Pregnancy, Amniotic fluid index (AFI), Congenital anomaly.

\begin{abstract}
A B S T R A C T
Background: Polyhydramnios is defined as a pathological increase of amniotic fluid volume in pregnancy. Common causes of polyhydramnios include gestational diabetes, fetal anomalies with disturbed fetal swallowing of amniotic fluid, fetal infections and others. The diagnosis is obtained by ultrasound. The prognosis of polyhydramnios depends on its cause and severity. Typical symptoms of polyhydramnios include maternal dyspnea, preterm labor, premature rupture of membranes (PPROM), abnormal fetal presentation, cord prolapsed, postpartum hemorrhage etc. Aim: Study was conducted to observe fetal outcome and related factors in polyhydramnios. Material and Methods: We studied 60 clinically suspected cases of polyhydramnios of more than 28 weeks of gestational age at Govt. Medical College Akola Maharashtra. All these cases were subjected to ultrasonography for fetal surveillance and AFI calculation. Result: The study revealed that predisposing factors and complications associated with polyhydramnios were preeclampsia, PROM, preterm labour, abruption placentae, malpresentation. There were maternal high risk factors seen was seen with anemia, Rh factor incompatibility, PIH, gestational diabetes mellitus and multifetal gestation. Polyhydramnios cases also had high proportion of central nervous system anomalies. Conclusion: We concluded from this study that there was higher proportion of congenital anomalies associated with polyhydramnios hence it is essential to evaluate amniotic fluid volume during antepartum fetal surveillance, while maternal examination should look for high risk factors. Thus management of women with polyhydramnios should be indivisualised with careful monitoring during labour, judicious and active intervention timely gives margin of safety.
\end{abstract}

Copyright $\bigcirc 2017$ Neethika Raghuwanshi and Sumit Suresh Aggarwal. This is an open access article distributed under the Creative Commons Attribution License, which permits unrestricted use, distribution, and reproduction in any medium, provided the original work is properly cited.

\section{INTRODUCTION}

Polyhydramnios is the term used to describe an excess accumulation of amniotic fluid. This clinical condition is associated with a high risk of poor pregnancy and fetal outcomes. The reported prevalence of polyhydramnios ranges from 0.2 to $1.6 \%$ of all pregnancies. ${ }^{[1]}$ Appropriate amount of amniotic fluid is essential for development of fetus. Any changes in normal course of fetus development can decrease or increase amniotic fluid, which will affect the outcome of pregnancy. ${ }^{[2]}$ The prevalence of polyhydramnios is increased in high-risk groups, such as those with maternal diabetes, placental diseases (e.g. chorioangioma) and various fetal abnormalities. ${ }^{[3]}$ Under physiological conditions there is a dynamic equilibrium between the production and resorption of amniotic fluid. Fluid levels are influenced by fetal urination and fetal lung liquid production. Amniotic fluid is reabsorbed by fetal swallowing and intramembranous and intravascular

*Corresponding author: Neethika Raghuwanshi

Department of Obstetrics \& Gynecology, Govt Medical College, Akola absorption. The relative attribution of each of these mechanisms varies over the course of the pregnancy. A disturbed equilibrium can be the result of compromised swallowing function or increased urination and can lead to polyhydramnios. ${ }^{[1]}$

In the present era of ultrasonography estimation of amniotic fluid volume (AFV) has replaced the subjective assessment of liquor amnii. Manning and platt in $(1980)^{[4]}$ defined normal amniotic fluid volume as maximum vertical pocket (MVP) of $<1 \mathrm{~cm}$, but this definition was too restrictive. Manning redefined normal AFV as single MVP of $2 \mathrm{~cm}$ in two perpendicular planes. Chamberlain and associates (1984) ${ }^{[5]}$ studied 7562 third trimester pregnant women and summarized criteria for AFV. Phelan and co-workers in $1987^{[4]}$ defined Amniotic Fluid Index (AFI) as the method of summing the MVP from each quadrant formed by drawing two perpendicular lines passing through umbilicus over the enlarged uterus on abdomen. So the present study was conducted to observe the fetal outcome in the case of polyhydramnios. 


\section{MATERIAL AND METHODS}

The present cross sectional observational study was conducted in the department of obstetrics and gynecology at Government Medical College, Akola, Maharashtra. The study duration was from $1^{\text {st }}$ September, 2015 to $31^{\text {th }}$ December, 2015 (total 4 months). Sampling method was universal sampling; all clinically suspected cases of polyhydramnios in third trimester were included in study. The study comprised of 60 clinically suspected cases of polyhydramnios in third trimester $(>28$ weeks of gestation) who presented in the Out Patient Department of obstetrics and gynecology.

Patients' detailed history was taken on standard Paper questionnaire, clinical examinations was done and positive findings were noted, routine and specific investigations were performed. Each and every patient was goes through detailed ultrsonography. Each patient was scanned in recumbent position with linear probe $5.5 \mathrm{MHz}$ transducer and assessment of amniotic fluid volume was done by four quadrant technique as described by Phelan et. al. (1987) ${ }^{[6]}$ known as amniotic fluid index. To measure AFI the landmark of the maternal abdomen were used to divide the uterine cavity into four sections; the transverse line drawn from umbilicus divides the abdomen in to upper and lower halves while lineanigra divides it into right $\&$ left halves. The transducer head is placed on maternal abdomen along the longitudinal axis and the MVP is measured, the number obtained from each quadrant was summed to calculate AFI. Depending upon the AFI levels the cases classified and managed. Informed verbal consent was taken from study subjects. Open source statistical software was used for statistical analysis.

\section{RESULT}

Table 1 Distribution of Study Subjects $(n=60)$ by Amniotic Fluid Volume by AFI

\begin{tabular}{ccc}
\hline AFV & AFI in cm & No. of patients (\%) \\
\hline Normal & $8.1-25 \mathrm{~cm}$ & $30(50)$ \\
Mild Increased & $25-30 \mathrm{~cm}$ & $10(16.7)$ \\
Moderately Increased & $30-35 \mathrm{~cm}$ & $16(26.7)$ \\
Severely Increased & $>35 \mathrm{~cm}$ & $04(06.6)$ \\
\hline
\end{tabular}

Out of 60 cases of suspected polyhydramnios, 50\% cases were to be in normal range of AFI, $16.7 \%$ cases had mild polyhydramnios, $26.7 \%$ cases were having moderate polyhydramnios and $06.6 \%$ cases had severe polyhydramnios.

Table 2 Distribution of Study Subjects according to their parity and AFI (in CM)

\begin{tabular}{cccccc}
\hline Parity & $\mathbf{8 . 1 - 2 5}$ & $\mathbf{2 5 . 1 - 3 0}$ & $\mathbf{3 0 . 1 - 3 5}$ & $>\mathbf{3 5}$ & Total (\%) \\
\hline P0 & 12 & 04 & 07 & 02 & $25(41.7)$ \\
P1 & 12 & 04 & 05 & 01 & $22(36.7)$ \\
P2 & 04 & 02 & 03 & 01 & $10(16.7)$ \\
P3 & 02 & 00 & 01 & 00 & $03(05)$ \\
Total & $30(50 \%)$ & $10(16.7 \%)$ & $16(26.7)$ & $04(6.6 \%)$ & $60(100)$ \\
\hline
\end{tabular}

Maximum number of study subjects was observed with parity P0 (41.7\%), then parity P1 (36.7\%), then parity P2 (16.7\%) and followed by parity P3 (05\%). AFI $30.1-35 \mathrm{~cm}$ was the most common situation in polyhydramnios irrespective of parity.

From the table no. 3 it was observed that Preterm labour (23.3\%), PROM (13.3\%), malpresentation (10\%) was most common conditions which was found to be associated with patients having AFI more than $35 \mathrm{~cm}$. Preterm labour was the most common morbidity which was associated with all grades of polyhydramnios.

Table 3 Perinatal Outcome associated with polyhydramnios $(n=30)$

\begin{tabular}{ccccc}
\hline Maternal & \multicolumn{3}{c}{ AFI(cm) } & Total \\
\cline { 2 - 4 } Factors & $\mathbf{2 5 - 3 0}$ & $\mathbf{3 0 - 3 5}$ & $>\mathbf{3 5}$ & $(\mathbf{\% )}$ \\
\hline Preterm labour & 01 & 02 & 04 & $07(23.3)$ \\
PROM & 00 & 01 & 03 & $04(13.3)$ \\
Malpresentation & 00 & 01 & 02 & $03(10.0)$ \\
Pre eclampsia & 00 & 02 & 00 & $02(06.6)$ \\
\hline
\end{tabular}

Table 4 Various pregnancy related factors associated with polyhydramnios $(n=30)$

\begin{tabular}{lcc}
\hline & Various factors & No of patients $(\%)$ \\
\hline & Anemia & $22(73.3)$ \\
a. & PIH & $18(60)$ \\
& Hypertational & $16(53.3)$ \\
b. $\quad$ Preeclampsion & $02(06.6)$ \\
Multiple gestation(Twins) & $06(20)$ \\
RH factor incompatibility & $02(06.6)$ \\
& Diabetes & $01(03.3)$ \\
\hline
\end{tabular}

It was observed from above table that The Polyhydramnios was found to be associated with Anemia (73.3\%) and PIH (60\%) most commonly followed by multiple gestation (twins) pregnancy (20\%) and Rh factor incompatibility (06.6\%).

Table 5 AFI and pregnancy outcome

\begin{tabular}{ccccc}
\hline Outcome & $\begin{array}{c}\mathbf{8 . 1 - 2 5} \\
(\mathbf{n = 3 0})\end{array}$ & $\begin{array}{c}\mathbf{2 5 - 3 0} \\
(\mathbf{n}=\mathbf{1 0})\end{array}$ & $\begin{array}{c}\mathbf{3 0 - 3 5} \\
(\mathbf{n}=\mathbf{1 6})\end{array}$ & $\begin{array}{c}\mathbf{3 5} \\
(\mathbf{n}=\mathbf{0 4})\end{array}$ \\
\hline $\begin{array}{c}\text { Caesarean section } \\
\text { Caesarean section for }\end{array}$ & 04 & 00 & 01 & 02 \\
FD & 02 & 00 & 00 & 00 \\
Apgar Score<7 & & & & \\
$-\quad$ 1 min & 00 & 00 & 00 & 02 \\
$-\quad$ 5 min & 00 & 00 & 00 & 00 \\
Meconium staining & 02 & 00 & 00 & 00 \\
Breech presentation & 02 & 00 & 01 & 02 \\
$\begin{array}{c}\text { Congenital } \\
\text { malformations }\end{array}$ & 00 & 02 & 05 & 02 \\
Preterm labour & 00 & 01 & 02 & 04 \\
Cord prolapsed & 00 & 00 & 01 & 01 \\
\hline
\end{tabular}

The table shows high rate of complication was observed in study subjects having AFI $>35 \mathrm{~cm}$ i.e. Preterm labour (100\%), LSCS $(50 \%)$, Breech presentation $(50 \%)$, congenital malformation (50\%) and Cord prolaps (25\%). Out 30 cases of polyhydramnios, $09(30 \%)$ cases of congenital malformation and 05 (16.7\%) cases of preterm labour was observed.

Table 6 Distribution of fetal congenital anomalies in polyhydramnios $(n=30)$

\begin{tabular}{ccc}
\hline Name of congenital anomaly & No. of cases (\%) & Perinatal Outcome \\
\hline $\begin{array}{c}\text { Urinary tract system(01) } \\
\text { a. Hydronephrosis }\end{array}$ CNS(05) & $1(03.33)$ & ENND \\
$\begin{array}{l}\text { a. Anencephaly } \\
\text { b. Spina bifida }\end{array}$ & $4(13.33)$ & MSB/ FSB/2 ENND \\
$\quad \begin{array}{l}\text { GI abnormalties(01) } \\
\text { a. Tracheo-oesophageal fistula }\end{array}$ & $1(03.33)$ & Alive \\
$\begin{array}{c}\text { Skeletal malformations }(03) \\
\text { a. Cleft palate with cleft lip } \\
\text { b. Cleft lip }\end{array}$ & $2(06.67)$ & FSB \\
Cardiac abnormalities(01) & $1(03.33)$ & Alive/ MSB \\
a. Pericardiac effusion & $1(03.33)$ & Alive \\
\hline
\end{tabular}

FSB* Fresh still birth, MSB* Macerated Still Birth, ENND* Early Neonatal Death

Above table shows higher incidence of congenital anomalies in central nervous system (16.67\%) fallowed by skeletal 
system $(10 \%)$ as compared to gastrointestinal (3.33), urinary system (3.33) and cardiovascular system (3.33).

Table 7 Analysis of perinatal mortality related to AFI

\begin{tabular}{ccccc}
\hline Fetal outcome & $\mathbf{8 . 1 - 2 5 c m}$ & $\mathbf{2 5 . 1 - 3 0 c m}$ & $\mathbf{3 0 - 3 5} \mathbf{~ c m}$ & $\mathbf{3 5 5} \mathbf{~ c m}$ \\
\hline Stillbirth & & & & \\
a. Fresh & 0 & 0 & 1 & 2 \\
b. Macerated & 1 & 0 & 0 & 1 \\
ENND & 0 & 1 & 2 & 0 \\
Total Perinatal mortality & $1(3.3)$ & $1(10)$ & $3(18.75)$ & $3(75)$ \\
$(\%)$ & & & & \\
\hline
\end{tabular}

The table 7 shows highest rate of Perinatal mortality in patient of severe polyhydramnios $(75 \%)$ fallowed by severe oligohydramnios $(25 \%)$ with minimum incidence in normal group (3.3\%).it shows that Perinatal mortality increases with increase in AFI.

\section{DISCUSSION}

Perinatal mortality is significantly increased when polyhydramnios is present at delivery. Fatal conditions that are associated with polyhydramnios are preeclampsia, PROM, preterm labour, abruption placentae and malpresentation. Accurate antepartum estimation of amniotic fluid volume by clinical means alone is exceedingly difficult but can be easily estimated by current diagnostic ultrasound methods (AFI).

Maternal factors found to be associated with polyhydramnios were Preeclampsia was noted in two cases of moderate polyhydramnios group, the incidence was $6.6 \%$. PROM was present in four cases out of which three cases were of severe polyhydramnios while one case was of moderate polyhydramnios. Preterm labour was found to be associated with seven cases $(23.3 \%)$ of polyhydramnios out of which maximum cases were in severe polyhydramnios. Perinatal mortality was increased further by preterm delivery even with normal fetuses. Many et. al. (1995) ${ }^{[7]}$ reported that $20 \%$ of 275 women with an AFI of $25 \mathrm{~cm}$ delivered preterm and it were more common with anomalous fetus $40 \%$. Malpresentation was seen in three cases $(10 \%)$ in this study two cases were seen in severe polyhydramnios one was breech and the other was compound presentation.

\section{Various pregnancy related factors associated}

Anemia was associated with 22 patients of polyhydramnios $(73.3 \%)$ as the rate of anemia is high in developing countries so the association was also high. $\mathrm{Rh}$ Factor: Negetive $\mathrm{Rh}$ factor was seen in two cases $(6.6 \%)$ but it was mere association the fetuses were normal without any evidence of immune hydrops. Pregnancy induced hypertension was noted in eighteen cases, sixteen patients $(53.3 \%)$ had gestational hypertention while two patients $(6.6 \%)$ had preeclampsia. Diabetes leads to third trimester polyhydramnios due to osmotic dieresis caused by fetal hyperglycemia. In our study we had one case of diabetes associated with moderate polyhydramnios. Multifetal gestation was seen in six patients all with twin pregnancy $(20 \%)$ all having moderate polyhydramnios.

\section{Pregnancy outcome in polyhydramnios}

In our study the proportion of preterm labour and malpresentation was high but the proportion of caesarean section was not increased. There were two patients $(50 \%)$ of breech presentation in severe polyhydramnios and one patient $(6 \%)$ in moderate polyhydramnios.
The incidence of preterm labour was high in polyhydramnios, maximum four cases in severe polyhydramnios $(100 \%)$ as compare to two $(13 \%)$ from moderate polyhydramnios and one $(10 \%)$ from mild polyhydramnios. There was no incidence of abruption placentae, postpartum hemorrhage and meconium staining.

\section{Perinatal outcome in polyhydramnios}

In the present study of 30 cases of polyhydramnios we had 11 (36.67\%) congenital malformations out of which 5 cases were of CNS malformations. Among these 5 cases one had spinabifida, baby was alive, the rest 4 had anencephaly one was macerated still birth second was fresh still birth and the other two had ENND within few hours.

There was one case $(3.33 \%)$ of urinary tract malformation. The fetus had bilateral hydronephrosis, the baby was alive at birth was admitted in nursery and had early neonatal death. One patient $(3.33 \%)$ had the gastrointestinal abnormality that was tracheo-osophageal fistula the baby delivered as fresh still births.

There were total three cases $(10 \%)$ of the skeletal malformation. Two (6.67\%) patients had cleft palate with cleft lip one was alive and the other was macerated still births. One baby had only cleft lip was alive. One (3.33\%) had pericardial effusion and delivered as fresh still birth.

Damato et al (1993) ${ }^{[8]}$ reported results from 105 women referred for evaluation of excessive fluid. Using definitions similar to those described by Hill et al. (1987) ${ }^{[9]}$. These investigators observed the almost $65 \%$ of the 105 pregnancies were abnormal. There were 47 singleton with one or more anomalies, gastrointestinal (15), non immunehydrops (12), central nervous system (12), thoracic (9), skeletal (8), chromosomal (7) and cardiac (4). Among 19 twin pregnancies, only two were normal. Twelve of the remaining 17 had twin twin transfusion.

\section{Quantitative amniotic fluid volume and perinatal mortality}

In the present study there was high perinatal mortality in severe polyhydramnios (75\%) followed by very low AFI $(25 \%)$ as compared to normal AFI $(3.3 \%)$.

There was one perinatal death among 30 patients with normal AFI. There was no perinatal death related to the fetal congenital anomalies. There were 5 perinatal deaths in patients with low AFI, one fetal death was related to congenital anomalies. Four still births, all in normal fetuses, occurred in this group.

There were 10 perinatal deaths in patients with very low AFI. Four perinatal deaths occurred in fetuses that were structurally normal. The remaining six perinatal deaths were the result of congenital anomalies. There was one perinatal death $(3.3 \%)$ in the normal AFI range which was not associated with congenital malformation. However, the perinatal mortality in the mild polyhydramnios was $1(10 \%)$, the cause was congenital malformation.

Chamberlain et al (1984) ${ }^{[10]}$ has found significantly greater perinatal mortality rate in decreased amniotic fluid volume as compared to perinatal mortality rate in marginal amniotic fluid volume and even lower perinatal mortality rate in the normal amniotic fluid volume. He also found greater still births rate in decreased $\mathrm{AFV}$ as compared to marginal $\mathrm{AFV}$ 
and normal AFV.

\section{CONCLUSION}

We concluded from this study that there was higher incidence of congenital anomalies associated with severe polyhydramnios. Thus it is essential to evaluate amniotic fluid volume during antepartum fetal surveillance. In presence of polyhydramnios thorough evaluation of the gravida for hypertension, pre-eclampsia, diabetes, chronic abruption, premature rupture of membrane, drug intake should be done. Thorough fetal anatomic survey focusing central nervous system in polyhydramnios, so that the patient may counsel for early termination before viability and unnecessary caesarean section can be avoided for IUGR, breech presentation and cord prolepses.

These findings suggests that patients with very low AFI should be monitored carefully during labour, and active intervention for a very low AFI, probably allows margin of safety before a major risk of perinatal complications is incurred.

Management of a woman with polyhydramnios must be individualized. One should strongly consider delivery if the fetus is likely to be mature. In case of gross immaturity pregnancies should be managed on maternal indications only.

\section{Reference}

1. Hamza, D Herr E F Solomayer, G MeybergSolomayer, Polyhydramnios: Causes, Diagnosis and Therapy. Geburtshilfe Frauenheilkd. 2013 Dec; 73(12): 1241-1246.

2. Neethika Raghuwanshi, Sumit S Aggarwal, a study of fetal outcome in pregnancies with inadequacy of liquor amnii at tertiary care center: a cross sectional study, EJBPS, 2017, Volume 4, Issue 1, 310-314.
3. N. Idris, S. F. Wong, M. Thomae, G. Gardener, D. H. McIntyre, Influence of polyhydramnios on perinatal outcome in pregestational diabetic pregnancies, Ultrasound Obstet Gynecol. 2010 Sep; 36(3): 338343. doi: 10.1002/uog.7676

4. Manning FA; Platt LD; Sipos J.: Antepartum fetal evaluation. Development of a fetal biophysical profile. Am J ObstGynecol 136:787-795/ 1980.

5. Chamberlain PF; Manning FA; Morrison I, et al: Ultrasound evaluation of amniotic fluid volume: I The relationship of marginal and decreased amniotic fluid volumes to perinatal outcome. Am. J. ObstetGynecol 150: $245-254 / 1984$

6. Phelan JP, Smith CV, Broussard P, Small M: Amniotic fluid volume assessment with the four-quadrant technique at 36-42 weeks' gestation. J Reprod Med 1987a; 32:540-542.

7. Many A, Hill LM, Lazbnik N, Martin JG: The association between polyhydramnios and preterm delivery. Obstet Gynecol 86:389, 1995.

8. Damato N, Filly RA, Goldstein RB, Callen PW, Goldberg J, Golbus M: Frequency of fetal anomalies in sonographically detected polyhydramnios. $J$ Ultrasound Med 1993;12:11

9. Hill LM, Breckle R, Thomas ML, Fries JK: Polyhydramnios ultrasonically detected prevalence and neonatal outcomes Obstet Gyneco1 69:21, 1987.

10. Chamberlain PF; ManningFA; Morrison I, et al: Ultasound evaluation of amniotic fluid volume: I the relationship of marginal and decreased amniotic fluid volumes to perinatal outcome. Am. J. Obstet Gynecol 150:245-254/1984.

\section{How to cite this article:}

Neethika Raghuwanshi and Sumit Suresh Aggarwal (2017) 'A Cross Sectional Study Of Fetal Outcome In Cases Of Polyhydramnios At Tertiary Care Center', International Journal of Current Advanced Research, 06(05), pp. 3960-3963. DOI: http://dx.doi.org/10.24327/ijcar.2017.3963.0406 